\title{
ACCIDENTE CEREBROVASCULAR Y FIBRILACIÓN AURICULAR EN UNA INSTITUCIÓN PRESTADORA DE SALUD DEL QUINDÍO
}

\author{
STROKE AND ATRIAL FIBRILLATION IN A HEALTH INSTITUTION OF QUINDÍO
}

Daniel Alberto Tabares-Montoya ${ }^{1}$; Stefany Barahona-Giraldo ${ }^{1}$; Lucy Ramírez-Reyes ${ }^{1}$;

Olga Alicia Nieto-Cárdenas $2 *$ (D).

1. Programa de Medicina. Facultad de Ciencias de la Salud. Grupo en Salud Pública. Universidad del Quindío.

2. Programa de Medicina. Grupo de Salud Pública. Facultad de Ciencias de la Salud. Universidad del Quindío. oanieto@uniquindio.edu.co

*Correspondencia del Autor: Olga Alicia Nieto-Cárdenas Universidad del Quindío. Programa de Medicina, correo electrónico: olgalicianieto@gmail.com.

\section{RESUMEN}

Introducción: La Fibrilación Auricular (FA) se ha relacionado frecuentemente con el Accidente Cerebrovascular (ACV). Objetivo: Describir la prevalencia de FA y sus comorbilidades en pacientes de un programa de Riesgo Cardiovascular de una Institución Prestadora de Servicios de Salud (IPS) del Quindío. Material y método: Estudio descriptivo de corte transversal. Se accedió a las historias clínicas de 193 pacientes con diagnóstico de FA, con consentimiento institucional. Se obtuvo una muestra de 53 sujetos de estudio, calculada para población finita, ajustada por prevalencia, aleatorizada, con reemplazo sistemático, sino se tenía la información requerida. Se calculó prevalencia de FA y de Accidente Cerebrovascular isquémico (ACVi), el uso de medicamentos para control de frecuencia, control del ritmo y anticoagulantes, la escala CHA2-DS2-VASC y la clasificación del riesgo. Resultados: Se encontró prevalencia de FA del 5,01\% y de ACVi del 7,55\%. Índice de Masa Corporal (IMC) promedio de 27,44 ( $\pm 6,20)$. El carvedilol fue el medicamento más usado (50,94\%) para control de la frecuencia, la amiodarona el medicamento más usado (11,32\%) para control del ritmo y el rivaroxabán $(50,94 \%)$ como anticoagulante. Se identificó hipertensión en $100 \%$ de los pacientes, diabetes en 37,74 \% y enfermedad vascular en 32,98 \%, las cuales son variables de la escala CHA2-DS2-VASC con alta prevalencia esta población. Conclusiones: La prevalencia de FA fue de 5,01\% y la prevalencia de ACVi del 7,55\%. El puntaje promedio de la escala CHA2-DS2-VASC fue 4,26 y el 88,68\% de la población se encontró en riesgo alto de Accidente cerebrovascular.

Palabras clave: Accidente cerebro vascular; fibrilación auricular; riesgo cardiovascular.

Cómo citar:

Tabares-Montoya, Daniel Alberto; Barahona-Giraldo, Stefany; Ramírez-Reyes, Lucy; Nieto-Cárdenas, Olga Alicia. (2021). Accidente cerebrovascular y fibrilación auricular en una Institución Prestadora de Salud del Quindío. Revista de Investigaciones Universidad del Quindio, 33(1), 115-125. https://doi.org/10.33975/riuq. vol33n1.458 


\section{ABSTRACT}

Introduction: Atrial Fibrillation (AF) has been frequently related to Cerebrovascular Accident (CVA). Objective: To describe the prevalence of Atrial Fibrillation and its comorbidities in patients of a Cardiovascular Risk program of a health institution (IPS) in Quindío. Material and method: Descriptive cross-sectional study. The medical records of 193 patients with a diagnosis of atrial fibrillation were accessed, with institutional consent. A sample of 53 study subjects was obtained, calculated for a finite population, adjusted for prevalence, randomized, with systematic replacement, if the required information was not available. The prevalence of atrial fibrillation and ischemic stroke, the use of drugs for rate control, rhythm control and anticoagulants, the CHA2-DS2-VASC scale and the risk classification were calculated. Results: Atrial fibrillation prevalence of 5.01\% and ischemic stroke of 7.55\% were found. Average body mass index (BMI) of $27.44( \pm 6.20)$. Carvedilol was the most used drug (50.94\%) for frequency control, amiodarone the most used drug (11.32\%) for rhythm control, and rivaroxaban (50.94\%) as anticoagulant. Hypertension was identified in $100 \%$ of the patients, diabetes in $37.74 \%$ and vascular disease in $32.98 \%$, which are variables of the CHA2-DS2-VASC scale with a high prevalence in this population. Conclusions: The prevalence of atrial fibrillation was $5.01 \%$ and the prevalence of ischemic stroke was 7.55\%. The average score of the CHA2-DS2-VASC scale was 4.26 and 88.68\% of the population found in high risk for Ischemic Stroke.

Keywords: Stroke; Atrial fibrillation; Cardiovascular risk.

\section{INTRODUCCIÓN}

La FA es la taquiarritmia supraventricular sostenida más común en la práctica clínica con una prevalencia del 2 al 8\%, y es más frecuente en mayores de 70 años. En pacientes con falla cardiaca, Hipertensión Arterial (HTA), diabetes mellitus, enfermedad coronaria y cardiopatía estructural, la prevalencia es mayor, del 10 al $20 \%(1-4)$. Es una arritmia cardiaca, en la cual se presentan estímulos eléctricos desorganizados y de alta frecuencia, lo que condiciona contracciones inefectivas de la aurícula, e impide el adecuado vaciamiento en el ventrículo, con una estasis sanguínea en la orejuela, que predispone a la formación de trombos que pueden viajar por la circulación sistémica y llegar a los vasos sanguíneos cerebrales, ocluyéndolos y así compromete el flujo sanguíneo cerebral (5).

$\mathrm{Su}$ diagnóstico se realiza con un electrocardiograma, donde se encuentran pequeñas ondas fibrilatorias (ondas f), en torno de la línea de base, con frecuencias entre 350 y
600 por minuto. La clasificación de la FA depende de la duración del episodio, de su relación con otras patologías y de su relación específica con valvulopatía $(1-3,5)$. En la fisiopatología un factor muy importante es el cambio estructural que puede sufrir el miocardio como consecuencia de una patología, o por cambios fisiológicos de la edad. Puede haber focos ectópicos y focos iniciadores de las descargas anormales, en la mayoría de los casos se identifica un foco iniciador en las venas pulmonares (94\%) y con menor frecuencia en la vena cava superior y el seno coronario (5).

El tratamiento de la FA tiene tres objetivos, control de la frecuencia cardíaca, control del ritmo cardíaco y la anticoagulación, que pretenden prevenir la aparición de nuevos episodios arrítmicos y del ACVi como complicación de la FA. Para el control de la frecuencia, los medicamentos de elección son los betabloqueadores, teniendo en cuenta que pueden ser utilizados independientemente de la Fracción de Eyección del Ventrículo Izquierdo (FEVI), 
situación que limita el uso de bloqueadores de los canales de calcio. Para el control del ritmo, la amiodarona es el medicamento de elección, sin embargo, se dispone de otras opciones como propafenona y flecainida. Para el manejo anticoagulante, se debe evaluar el tipo de FA que se presenta, en el caso de FA valvular, se ve mayor beneficio con medicamentos antivitamina $\mathrm{K}$ como la warfarina, mientras que, en pacientes con FA no valvular, se observa mayor beneficio con anticoagulantes orales como el rivaroxabán, sin embargo, la decisión de la terapia anticoagulante depende de la valoración clínica del riesgo de eventos trombóticos, que puede ser realizada con escalas estandarizadas y validadas como la CHA2-DS2-VASC (2,5-7).

El ACVi representa la segunda causa de muerte, después de la enfermedad isquémica del corazón y equivale al 10,6\% de la mortalidad mundial. Y se encuentra en el tercer lugar de causa de Años de Vida Ajustados en función de la Discapacidad (AVAD). En Colombia, las enfermedades del sistema circulatorio fueron la primera causa de mortalidad en hombres y en mujeres; y según estudios llevados a cabo entre 2005 y 2014 se encuentran como primera causa de muerte las enfermedades del corazón y en segundo lugar las enfermedades cerebrovasculares; el ACVi representa el $70 \%$ de todos los ACV en Colombia (8-12).

El ACVi, es una afección neurológica focal, transitoria o permanente, que dura más de 24 horas (o causa la muerte), de presunto origen vascular, es causado por la oclusión súbita de los vasos arteriales que están encargados de la irrigación del cerebro, debido a un trombo, que puede formarse directamente en el lugar donde se generó la oclusión, denominándose ACVi trombótico, o por la formación de un trombo en otro sitio del sistema vascular, que viaja por el torrente sanguíneo, instaurándose en las arterias del cerebro, denominándose $\mathrm{ACVi}$ embólico, aunque la causa difícilmente puede ser definida $(9,13,14)$.
El tratamiento del ACVi tiene como objetivo la reperfusión de la zona de penumbra, una zona periférica a la lesión, en donde hay una disminución de la tasa metabólica por el bajo flujo sanguíneo, sin haber muerte celular, por lo que se puede recuperar con la reperfusión, pero se debe hacer de manera temprana, y después de un cuidadoso análisis del cuadro del paciente y las imágenes obtenidas por tomografía o resonancia, para descartar la presencia de un ACV hemorrágico, porque el tratamiento es diferente. La reperfusión se puede lograr con el uso de Activador de Plasminógeno Tisular (tPA), que induce la activación de plasminógeno en plasmina, lo que hidroliza la fibrina generando trombolisis. Adicionalmente, la terapia endovascular, una estrategia en la que se hace la remoción del trombo a través de un acceso arterial vía catéter, guiado por imagenología, aporta una opción terapéutica en pacientes en los que está contraindicada la trombolisis por factores particulares (10,13-16).

El ACVi, secundario a FA, se define como un problema debido a la alta carga de morbimortalidad que representa, porque condiciona pérdida de la funcionalidad temporal o permanente de los pacientes, lo cual se refleja en los AVAD, con las repercusiones económicas y sociales que esto trae consigo, porque este paciente no puede desempeñar con normalidad sus roles dentro de la sociedad, además, la afectación en la dinámica familiar que representa el cuidado de un paciente en estas condiciones. Es así como estos pacientes representan un alto costo para el sistema de salud, desde su atención inicial, costos de hospitalización y de rehabilitación integral, que deben permitir al paciente su reincorporación a la sociedad con la máxima funcionalidad posible $(11,12,17-19)$.

En el Departamento del Quindío se desconoce la prevalencia de la FA y su relación con el ACVi, por lo cual el presente trabajo pretende describir la prevalencia de FA en la población a estudio, identificar la prevalencia de ACVi en pacientes con FA en esta población y describir la relación de la prevalencia de ACVi y FA en la población 
del departamento del Quindío, que consultó al programa de Riesgo Cardiovascular (RCV) de una IPS del Quindío.

\section{METODOLOGÍA}

Estudio descriptivo, de corte transversal, con un universo (N) de 193 historias clínicas de pacientes con diagnóstico de FA, consultantes al programa de RCV de una IPS del departamento del Quindío (Fundación Participar), una muestra (n) de 53, obtenida con fórmula para poblaciones finitas ajustada por prevalencia, aleatorizada en Excel ${ }^{\circledR} 2016$, con reemplazo sistemático de las historias clínicas que no cumplían con los criterios de inclusión y exclusión. Esta población es de pacientes remitidos del Quindío y norte del Valle del Cauca, lo que permite una diversidad demográfica que enriquece el estudio.

Criterios de inclusión: Pacientes que consultan a un programa de RCV de una IPS del Quindío con diagnóstico de FA registrado en su historia clínica.

Criterios de exclusión: Pacientes que hayan presentado accidente cerebro vascular hemorrágico y pacientes menores de 18 años.

Se registró la información de las variables obtenidas de las historias clínicas, en una base de datos en Excel® 2016, para luego ser analizadas en Statghaphics Centurion XVII ${ }^{\circledR}$ mediante pruebas de normalidad, medidas de tendencia central, análisis de varianza (ANOVA) para las variables numéricas y una prueba de Chi cuadrado para las variables categóricas. Se realizaron análisis diferenciales de cada variable por sexo y por diagnóstico previo de ACVi.

El proyecto tiene aprobación del comité de bioética de la Facultad de Ciencias de la Salud de la Universidad del Quindío, mediante el Acta No. 08 (13 de diciembre de 2017) y se gestionó el consentimiento institucional con la IPS, bajo el amparo del decreto 1377 de 2013, el cual reglamenta el uso de la información sensible.

\section{RESULTADOS}

Se encontró una prevalencia de FA del 5,01\% en los pacientes que consultaron al programa de riesgo cardiovascular en una IPS del departamento del Quindío en el año 2017.

El promedio de edad fue de 77,56 \pm 8,59 años, con un peso promedio de $67,11 \pm 16,09 \mathrm{~kg}$, y una talla promedio de $1,56 \pm 0,08 \mathrm{~m}$, con un IMC promedio de $27,44 \pm 6,20$ es decir en sobrepeso (Tabla 1).

Con respecto a la escala CHA2-DS2-VASC, encontramos que el $100 \%$ de la población estudiada tenía registrada en la historia clínica dicha escala; con un promedio para un puntaje total de la escala de 4,26 $\pm 1,42$ dejando a la mayoría en una clasificación de riesgo alto; con una distribución por sexo de 3,72 $\pm 1,30$ para los hombres y de 4,75 $\pm 1,37$ para mujeres, aunque la mayoría de los pacientes se clasifica en riesgo alto, las mujeres presentan un puntaje mayor; con una diferencia estadísticamente significativa (valor de $\mathrm{p}<$ de 0,01 ) (Tabla 1).

La prevalencia de ACVi fue de 7,55\%. 49 pacientes $(92,45 \%)$ no tenían el diagnóstico de $\mathrm{ACVi}$, mientras 2 pacientes uno femenino y uno masculino $(3,77 \%)$ tenían diagnóstico de accidente vascular encefálico agudo no especificado como hemorrágico o isquémico, 1 paciente femenino $(1,89 \%)$ tenía diagnóstico de ataque de isquemia cerebral transitoria y síndromes afines y 1 paciente masculino $(1,89 \%)$ con diagnóstico de infarto cerebral no especificado (Tabla 2).

Recibían tratamiento para el control de la frecuencia cardiaca 37 pacientes $(69,81 \%)$, de los cuales 15 pacientes son masculinos y 22 femeninos; en cuanto al tipo de medicamento para el control de la frecuencia el carvedilol fue el medicamento más usado $(50,94 \%)$, seguido en orden por metoprolol $(7,55 \%)$, amiodarona $(7,55 \%)$ y verapamilo $(3,77 \%)$. En los pacientes con diagnóstico de ACVi, la mitad (2 pacientes), estaban recibiendo tratamiento para el control de 
la frecuencia, el carvedilol fue el medicamento formulado en estos pacientes (Tabla 2).

Para el control del ritmo, 45 pacientes $(84,91 \%)$ no recibían tratamiento, dentro de los cuales se encuentran los 4 pacientes con diagnóstico de ACVi; mientras 8 pacientes $(15,09 \%), 5$ femeninos y 3 masculinos si lo recibían: 6 pacientes, 3 masculinos y 3 femeninos $(11,32 \%)$ recibían amiodarona, 1 paciente femenino $(1,89 \%)$ recibía betametildigoxina y 1 paciente femenino (1,89\%) digoxina (Tabla 2 ).

Del tratamiento anticoagulante, 2 pacientes $(3,77 \%)$ que tenían el diagnóstico de $\mathrm{ACV}$ recibieron este tipo de tratamiento con rivaroxabán, y los otros dos casos con el diagnóstico no lo estaban recibiendo. Es decir, $50 \%$ de los pacientes con diagnóstico de ACV no estaban recibiendo tratamiento anticoagulante. De los pacientes sin diagnóstico de ACV, 47,17\% recibió rivaroxabán, $7,55 \%$ warfarina, $5,66 \%$ dabigatrán, 3,77\% apixabán, y 28,30\% no lo estaban recibiendo (Tabla 2).

En la clasificación del riesgo no se encontraron pacientes con riesgo bajo, el 11,32\% tenían riesgo moderado y el 88,68 \% presentaban riesgo alto; de los cuales el $37,74 \%$ son pacientes masculinos y el $50,94 \%$ son pacientes femeninos, con diferencia estadísticamente significativa (valor de $\mathrm{p}=0,05)$ (Tabla 3).

Al evaluar las variables que componen la escala CHA2-DS2-VASC, se encontró que las que más relevancia tienen en la población estudiada son HTA, diabetes mellitus y enfermedad vascular.

El diagnóstico de HTA estaba presente en el $100 \%$ de los pacientes. La prevalencia de diabetes mellitus fue de 37,74\% del total de la muestra de la población. De los cuales 2 pacientes con diabetes mellitus tiene diagnóstico de ACV. Con respecto a la enfermedad vascular periférica, se encontró una prevalencia del $32,08 \%$ en pacientes con FA, estando presente en todos los pacientes con diagnóstico de $\mathrm{ACVi}$ con una diferencia estadísticamente significativa (valor de $\mathrm{p}<0,01$ ), (Tabla 4).

\section{DISCUSIÓN}

Se encontró una prevalencia de ACV en pacientes con FA del $5,01 \%$ en la población de estudio, información que es importante porque no se tienen registros previos en el departamento del Quindío. En otro estudio en Colombia en 3 instituciones, se encontró una prevalencia global del 4,8\%, que aumenta 3,3 veces al pasar los 70 años, hecho que demuestra que en Colombia esta enfermedad crece a mayor rango etario, datos útiles para sustentar políticas de tamizaje, que mejoren la calidad y el cubrimiento en salud (4).

Se identificó que la prevalencia de $\mathrm{ACV}$ en la población del estudio fue de $7,5 \%$, pero no se encontraron datos de prevalencia de ACV en pacientes con FA en el Quindío, lo que no permite confrontar los resultados obtenidos en este estudio.

En cuanto a los factores de riesgo asociados al aumento de prevalencia de ACV en esta población, se encontró que:

La HTA estuvo presente en el $100 \%$ de los pacientes del estudio, superior a la prevalencia encontrada en estudio ATHENA 2009 (20), que identificó una prevalencia del $83 \%$ en pacientes con FA.

Se identificó una prevalencia de diabetes mellitus del 37,74\% del total de la muestra de la población, que es superior a la prevalencia descrita en Colombia por Bonilla et al, 2014 (21), del 25,5\% para población general.

Con respecto a la enfermedad vascular periférica, en este estudio se halló, una prevalencia del $32,08 \%$ en pacientes con FA, con una diferencia estadísticamente significativa entre pacientes con diagnóstico de ACVi y aquellos que no lo tenían. Huttner et al. 2010 (22), encontraron una mayor prevalencia de esta condición en 
pacientes con $\mathrm{ACVi}$, comparado con otros tipos como la isquemia cerebral transitoria y el ACV hemorrágico, sin embargo no lo relacionó con el diagnóstico de FA.

Este estudio muestra que la enfermedad vascular tiene relación con el ACV en esta población con FA, sin embargo, por las limitaciones propias del tipo de un estudio descriptivo con una muestra baja, no se puede hablar de una relación de causalidad. Lo anterior reafirma que esta variable tiene un papel importante como componente de la escala CHA2-DS2-VASC para predecir el riesgo de ACVi en esta población específica.

El promedio del puntaje de la escala de CHA2DS2-VASC fue de 3,72 $\pm 1,30$ puntos en hombres y 4,75 $\pm 1,37$ puntos en mujeres, con una diferencia estadísticamente significativa, que indica que las mujeres presentaban mayor riesgo de presentar un $\mathrm{ACV}$; además, las mujeres presentaron mayor porcentaje de riesgo severo $(50,94 \%)$ que los hombres $(37,74 \%)$. Aunque no hay datos estadísticos puntuales que sean diferenciales por sexo, según la American Heart Association y la American Stroke Association (AHA / ASA) 2015 (10), se reporta un mayor riesgo de eventos trombóticos en mujeres, relacionado con factores importantes como ciclos hormonales, métodos anticonceptivos, estilos de vida y cambios fisiológicos con la edad como la menopausia.

Sobre el uso de medicamentos para el control de la frecuencia, se identificó que el grupo de medicamentos más usado fueron los betabloqueadores, con el carvedilol como fármaco más utilizado en un 50,94\%; información similar se encuentra en España, según la Sociedad Española de Cardiología, en el registro FANTASIIA 2016 (23), se informa mayor uso de betabloqueadores como medicamento para este fin. Los betabloqueadores, según CadrinTourigny et al. 2017 (24), disminuyen la mortalidad por todas las causas, en pacientes con diagnóstico de FA y falla cardíaca.
En este estudio se encontró que el uso de medicamentos antiarrítmicos fue del 15,09\% y la amiodarona fue el medicamento más usado $(11,32 \%)$; lo cual está por debajo de otros estudios y seguimientos internacionales como el registro FANTASIIA 2016 (23), en el que se encontró que el uso de fármacos antiarrítmicos fue del 24,7\%, aunque coincide con la información referente a la amiodarona como el medicamento más utilizado.

Según la Sociedad Española de Cardiología, en el registro FANTASIIA 2016 (23), el mayor uso de los betabloqueadores sobre los antiarrítmicos, está relacionado con la evidencia de no superioridad de la terapia para control de ritmo cardíaco sobre la terapia para el control de la frecuencia, en la prevención del ACVi.

Se identificó en el estudio que, el rivaroxabán es el medicamento anticoagulante más usado (50,94\% de los pacientes). De acuerdo con la AHA y la ASA 2015 (10), los anticoagulantes orales tienen una mayor adherencia en los pacientes que otros como la warfarina, por la vía de administración y la menor tasa de incidencia de efectos adversos. Así mismo, Ruff et al. 2014 (25) encontraron una reducción mayor de ACV, embolia sistémica y mortalidad por todas las causas con fármacos anticoagulantes orales que con warfarina.

\section{CONCLUSIONES}

La prevalencia de FA en una IPS del Quindío fue del 5,01\%, lo cual es un aporte significativo al conocimiento de las características epidemiológicas de esta población y puede aportar para la planeación en salud pública de intervenciones en RCV; y la prevalencia de ACVi en la población estudiada, con diagnóstico de FA fue del 7,55\%, lo cual es un aspecto importante al ser un dato nuevo en el conocimiento de esta población.

La escala CHA2-DS2-VASC, como escala útil en la evaluación del riesgo de $\mathrm{ACV}$, permitió la identificación de algunas variables que 
tienen mayor relevancia en esta población, encontrándose un puntaje total mayor para las mujeres, que, para los hombres. Y los factores de riesgo para ACV identificados en pacientes con diagnóstico de FA en esta población, fueron la hipertensión, la enfermedad vascular y la diabetes.

En el manejo de la población estudiada, cobran especial interés tres categorías de medicamentos: el carvedilol como betabloqueador, la amiodarona para el control del ritmo y el rivaroxabán como anticoagulante.

\section{AGRADECIMIENTOS}

Agradecemos al Dr. Cesar Beltrán por su apoyo a este proceso investigativo y a las instituciones que hicieron posible este estudio, a la IPS Fundación Participar y a la Universidad del Quindío.

\section{REFERENCIAS}

1. Romero M, Chávez D. Carga de enfermedad atribuible a fibrilación auricular en Colombia (20002009). Rev Colomb Cardiol [Internet]. 2014 [citado el 1 de septiembre de 2017];21(6):374-81. Disponible en: http://dx.doi.org/10.1016/j.rccar.2014.08.006

2. Baena J, Benitez W, Bermudez J, Betancourt J, Cabrales M, Cardona H, et al. Guía de diagnóstico y tratamiento de la fibrilación auricular. Rev Colomb Cardiol [Internet]. 2007;14(Supl. 3):51-183. Disponible en: http://scc.org.co/wp-content/uploads/2012/08/6-fibrilacion-auricular-2007.pdf

3. Ramírez-Barrera JD, Agudelo-Uribe JF, Correa-Velásquez R, González-Rivera E. Fisiopatología de la fibrilación auricular. Rev Colomb Cardiol [Internet]. 2016;23 (Supl.5):8-15. Disponible en: http://cardiolatina.com/wp-content/uploads/2017/04/Guias-Colombianas-FA-SCC_CCE.pdf

4. Castro-Clavijo JA, Quintero S, Valderrama F, Diaztagle JD, Ortega J.Prevalencia de fibrilación auricular en pacientes hospitalizados por Medicina interna. Revista Colombiana de Cardiología. 2020; 27(69):557-63. Disponible en: https://www.sciencedirect.com/science/article/pii/ $\underline{\mathrm{S} 0120563319301044}$

5. EHRA/ESC. Guía ESC 2016 sobre el diagnóstico y tratamiento de la fibrilación auricular, desarrollada en colaboración con la EACTS. Rev Española Cardiol [Internet]. 2016 [citado el 7 de septiembre de 2017];70(1):1-84. Disponible en: http://www.siacardio.com/wp-content/ uploads/2015/01/Guias-Colombianas-FA-SCC_CCE.pdf

6. Uribe W, Medina E, Duque M. Fibrilación auricular : una mirada actual. Rev Colomb Cardiol [Internet]. 2012 [citado el 1 de septiembre de 2017];19(5):235-51. Disponible en: http://www. scielo.org.co/scielo.php?script=sci_arttext\&pid=S0120-56332012000500006

7. Lane DA, Lip GYH. Use of the CHA2DS2-VASc and HAS-BLED scores to aid decision making for thromboprophylaxis in nonvalvular atrial fibrillation. Circulation. 2012;126(7):860-5.

8. Organización Mundial de la Salud. Estrategia paso a paso de la OMS para la vigilancia de accidentes cerebrovasculares. In Geneve; 2005 [citado el 1 de septiembre de 2017]. p. 113. Disponible en: http://www1.paho.org/hq/dmdocuments/2009/manuales.pdf

9. Varela G. Enfermedad Cerebrovascular - Tratamiento del Ataque Cerebrovascular Isquemico Agudo. Acta Neurológica Colombiana [Internet]. 2009 [citado el 06 de septiembre de 2017]. p. 151-60. Disponible en: http://www.acnweb.org/es/publicaciones/guia-8-ecv/328-tratamiento-deataque-cerebrovascular-acv-isquemico-agudo.html

10. Powers WJ, Derdeyn CP, Biller J, Coffey CS, Hoh BL, Jauch EC, et al. 2015 American Heart Association/American stroke association focused update of the 2013 guidelines for the early management of patients with acute ischemic stroke regarding endovascular treatment: A guideline for healthcare professionals from the American Heart Association/American Stroke Association. 2015;46(10):3020-35.

11. Wang H, Naghavi M, Allen C, Barber RM, Carter A, Casey DC, et al. Global, regional, and national life expectancy, all-cause mortality, and cause-specific mortality for 249 causes of death, 1980-2015: a systematic analysis for the Global Burden of Disease Study 2015. Lancet [Internet]. 2016 [Consultado el 1 de septiembre de 2017];388(10053):1459-544. Disponible en: https://www. thelancet.com/pdfs/journals/lancet/PIIS0140-6736(16)31012-1.pdf

12. Kassebaum NJ, Arora M, Barber RM, Brown J, Carter A, Casey DC, et al. Global, regional, and 
national disability-adjusted life-years (DALYs) for 315 diseases and injuries and healthy life expectancy (HALE), 1990-2015: a systematic analysis for the Global Burden of Disease Study 2015. Lancet [Internet]. 2016 [citado el 1 de septiembre de 2017];388(10053):1603-58. Disponible en: https://www.thelancet.com/pdfs/journals/lancet/PIIS0140-6736(16)31012-1.pdf

13. Powers WJ, Derdeyn CP, Biller J, Coffey CS, Hoh BL, Jauch EC, et al. 2015 American Heart Association/American Stroke Association Focused Update of the 2013 Guidelines for the Early Management of Patients With Acute Ischemic Stroke Regarding Endovascular Treatment. Stroke [Internet]. 2015 [citado el 1 de septiembre de 2017];46(10). Disponible en: http://stroke. ahajournals.org/content/46/10/3020

14. James SK, Stenestrand U, Lindback J, Carlsson J, Schersten F, Nilsson T, et al. Thrombolysis with Alteplase 3 to 4.5 Hours after Acute Ischemic Stroke. N Engl J Med [Internet]. 2009 [citado el 1 de septiembre de 2017] ;360(19):1933-11945. Disponible en: https://www.nejm.org/doi/ full/10.1056/NEJMoa0804656

15. Collazos MM, Gutiérrez ÁM, Londoño D, Bayona H, Herrán S, Enrique G. Uso del Activador de Plasminógeno Tisular Recombinante (rt-PA) en el Ataque Cerebrovascular isquémico en Colombia: Un estudio de costo-efectividad. Tissue plasminogen activator (rt-PA) for acute ischemic stroke in Colombia: a cost-effectiveness study. Acta Neurol Colombiana [Internet]. 2008 [citado el 7 de septiembre de 2017];24:158-73. http://www.acnweb.org/acta/2008_24_4_158.pdf

16. Campbell BCV, Mitchell PJ, Kleinig TJ, Dewey HM, Churilov L, Yassi N, et al. Endovascular Therapy for Ischemic Stroke with Perfusion-Imaging Selection. N Engl J Med [Internet]. 2015 [citado el 7 de septiembre de 2017];372(11):1009-18. Disponible en: http://www.nejm.org/ doi/10.1056/NEJMoa1414792

17. Flores G E, Rivas R E, Seguel P F. Nivel de sobrecarga en el desempeño del rol del cuidador familiar de adulto mayor con dependencia severa. Cienc y enfermería [Internet]. 2012 Apr [citado el 07 de septiembre de 2017];18(1):29-41. Disponible en: http://www.scielo.cl/scielo.php?script=sci_ arttext\&pid=S0717-95532012000100004\&lng=en\&nrm=iso\&tlng=en

18. Jofré Aravena V, Sanhueza Alvarado O. Evaluación de la sobrecarga de cuidadoras/es informales. Ciency enfermería [Internet].2010 Dec [citado el 7 de septiembre de 2017];16(3):111-20. Disponible en: http://www.scielo.cl/scielo.php?script=sci_arttext\&pid=S0717-95532010000300012\&lng=en $\&$ nrm=iso\&tlng=en

19. Luengo C, Araneda G, López M. Factores del cuidador familiar que influyen en el cumplimiento de los cuidados básicos del usuario postrado. Index de Enfermería [Internet]. 2010 [citado el 7 de septiembre de 2017];19(1):14-8. Disponible en: http://scielo.isciii.es/scielo.php?pid=S1132$12962010000100003 \&$ script=sci_arttext\&tlng=en

20. Pikto-Pietkiewicz W. The effect of dronedarone on the frequency of cardiovascular events in patients with atrial fibrillation - ATHENA studies. Kardiol Pol. 2009;67(4):455-6.

21. Bonilla S NP, Oliveros H, Proaños J, Espinel B, Álvarez JC, Duran C, et al. Estudio de Frecuencia de los Factores de Riesgo Asociados al Desarrollo de Enfermedad Cerebrovascular Isquémica No Embólica en un Hospital de Tercer Nivel. Acta Neurológica Colomb [Internet]. 2014;30(3):14955. Disponible en: http://www.scielo.org.co/scielo.php?script=sci_arttext\&pid=S0120$87482014000300004 \& \operatorname{lng}=$ en\&nrm=iso\&tlng=es

22. Huttner HB, Köhrmann M, Mauer C, Lücking H, Kloska S, Doerfler A, et al. The prevalence of peripheral arteriopathy is higher in ischaemic stroke as compared with transient ischaemic attack and intracerebral haemorrhage. Int J Stroke. 2010;5(4):278-83.

23. Sa MA, Marı F, Siles C, Peinado R, Bertomeu V, Fillat AC, et al. Current Antiarrhythmic Therapy for Nonvalvular Atrial Fibrillation in Spain . Data From the FANTASIIA Registry ${ }^{\sim}$ iz , on behalf of the FANTASIIA study researchers. Rev Esp Cadiologia 2016;69(1):54-60.

24. Cadrin-tourigny J, Shohoudi A, Roy D, Talajic M, Dyrda K, Andrade JG, et al. Decreased Mortality With Beta-Blockers in Patients With Heart Failure and Coe xisting Atrial Fibrillation. JACC heart fail 5/02/2017.25.

25. Ruff CT, Giugliano RP, Braunwald E, Hoff EB, Deenadayalu N, Ezekowitz MD, et al. Comparison of the efficacy and safety of new oral anticoagulants with warfarin in patients with atrial fibrillation : LANCET. 2014;955-62. 
TABLAS

Tabla 1. Variables numéricas por sexo.

\begin{tabular}{|c|c|c|c|c|c|c|c|}
\hline Variable & $\mathbf{X} \pm \mathbf{S}$ & IC $95 \%$ & Masc & & Feme & & $\begin{array}{l}\text { Valor de p } \\
\text { (ANOVA) }\end{array}$ \\
\hline Edad (años) & $77,56 \pm 8,59$ & $75,19-79,93$ & 76,72 & $\pm 8,99$ & 78,32 & $\pm 8,30$ & 0,50 \\
\hline Peso (kg) & $67,11 \pm 16,09$ & $62,67-71,54$ & 70,72 & $\pm 14,72$ & 63,89 & $\pm 16,83$ & 0,12 \\
\hline Talla (m) & $1,56 \pm 0,08$ & $1,53-1,58$ & 1,61 & $\pm 0,06$ & 1,51 & $\pm 0,07$ & $<0,01$ \\
\hline IMC & $27,44 \pm 6,20$ & $25,73-29,15$ & 27,03 & $\pm 5,33$ & 27,81 & $\pm 6,97$ & 0,65 \\
\hline $\begin{array}{l}\text { Frecuencia } \\
\text { cardiaca (latidos } \\
\text { por minuto) }\end{array}$ & $75,92 \pm 14,06$ & $72,04-79,80$ & 74,04 & $\pm 14,24$ & 77,60 & $\pm 13,94$ & 0,36 \\
\hline $\begin{array}{l}\text { Escala CHA2- } \\
\text { DS2-VASC } \\
\text { (puntaje total) }\end{array}$ & $4,26 \pm 1,42$ & $3,87-4,65$ & 3,72 & $\pm 1,30$ & 4,75 & $\pm 1,37$ & $<0,01$ \\
\hline
\end{tabular}

Tabla 2. Variables categóricas por sexo.

\begin{tabular}{|c|c|c|c|c|c|c|c|c|}
\hline \multirow[t]{2}{*}{ Variable } & \multirow[t]{2}{*}{ Categorías } & \multicolumn{2}{|c|}{ Masculino } & \multicolumn{2}{|l|}{ Femenino } & \multicolumn{2}{|l|}{ Total } & \multirow{2}{*}{$\begin{array}{l}\text { Valor de } p \\
\left(\begin{array}{c}C h \\
\text { cuadrado) }\end{array}\right.\end{array}$} \\
\hline & & $\begin{array}{l}\text { Numero } \\
\mathrm{n}: 25\end{array}$ & $\begin{array}{l}\text { Porcen } \\
\text { aje \% }\end{array}$ & $\begin{array}{l}\text { Numero } \\
\mathrm{n}: 28\end{array}$ & $\begin{array}{l}\text { Porcentaje } \\
\%\end{array}$ & $\begin{array}{l}\text { Numero } \\
\text { n: } 53\end{array}$ & $\begin{array}{l}\text { Porcentaje } \\
\%\end{array}$ & \\
\hline \multirow[t]{4}{*}{$\begin{array}{l}\text { Diagnóstico } \\
\text { ACV CIE } 10\end{array}$} & $\begin{array}{l}\text { Accidente vascular } \\
\text { encefálico agudo } \\
\text { no especificado } \\
\text { como hemorrágico } \\
\text { o isquémico }\end{array}$ & 1 & 1,89 & 1 & 1,89 & 2 & 3,77 & \multirow[t]{4}{*}{0,56} \\
\hline & $\begin{array}{l}\text { Ataques de } \\
\text { isquemia cerebral } \\
\text { transitoria y } \\
\text { síndromes afines }\end{array}$ & 0 & 0 & 1 & 1,89 & 1 & 1,89 & \\
\hline & $\begin{array}{l}\text { Infarto cerebral no } \\
\text { especificado }\end{array}$ & 1 & 1,89 & 0 & 0 & 1 & 1,89 & \\
\hline & $\begin{array}{l}\text { No tiene } \\
\text { diagnóstico de ACV }\end{array}$ & 23 & 43,40 & 26 & 49,06 & 49 & 92,45 & \\
\hline \multirow[t]{4}{*}{ EKG Onda P } & Onda P presente & 9 & 16,98 & 14 & 26,42 & 23 & 43,40 & \multirow[t]{4}{*}{0,40} \\
\hline & Onda $\mathrm{P}$ ausente & 11 & 20,75 & 7 & 13,21 & 18 & 33,96 & \\
\hline & Marcapasos & 0 & 0 & 1 & 1,89 & 1 & 1,89 & \\
\hline & No tiene EKG & 5 & 9,43 & 6 & 11,32 & 11 & 20,75 & \\
\hline \multirow[t]{4}{*}{ EKG Ritmo } & Ritmo sinusal & 9 & 13,98 & 16 & 30,19 & 25 & 47,17 & \multirow[t]{4}{*}{0,16} \\
\hline & Ritmo nodal & 11 & 20,75 & 5 & 9,43 & 16 & 30,19 & \\
\hline & Marcapasos & 0 & 0 & 1 & 1,89 & 1 & 1,89 & \\
\hline & No tiene EKG & 5 & 9,43 & 6 & 11,32 & 11 & 20,75 & \\
\hline \multirow{2}{*}{$\begin{array}{l}\text { Tratamiento } \\
\text { frecuencia }\end{array}$} & $\mathrm{Si}$ & 15 & 28,30 & 22 & 41,51 & 37 & 69,81 & \multirow[t]{2}{*}{0,14} \\
\hline & No & 10 & 18,87 & 6 & 11,32 & 16 & 30,19 & \\
\hline
\end{tabular}




\begin{tabular}{|c|c|c|c|c|c|c|c|c|}
\hline \multirow{5}{*}{$\begin{array}{l}\text { Medicamento } \\
\text { Frecuencia }\end{array}$} & Carvedilol & 10 & 18,87 & 17 & 32,08 & 27 & 50,94 & \multirow[t]{5}{*}{0,32} \\
\hline & Amiodarona & 3 & 5,66 & 1 & 1,89 & 4 & 7,55 & \\
\hline & Metoprolol & 1 & 1,89 & 3 & 5,66 & 4 & 7,55 & \\
\hline & Verapamilo & 1 & 1,89 & 1 & 1,89 & 2 & 3,77 & \\
\hline & $\begin{array}{l}\text { No tiene } \\
\text { tratamiento }\end{array}$ & 10 & 18,87 & 6 & 11,32 & 16 & 30,19 & \\
\hline \multirow{2}{*}{$\begin{array}{l}\text { Tratamiento } \\
\text { antiarrítmico }\end{array}$} & $\mathrm{Si}$ & 3 & 5,66 & 5 & 9,43 & 8 & 15,09 & \multirow[t]{2}{*}{0,55} \\
\hline & No & 22 & 41,51 & 23 & 43,40 & 45 & 84,91 & \\
\hline \multirow{4}{*}{$\begin{array}{l}\text { Medicamento } \\
\text { Antiarrítmico }\end{array}$} & Amiodarona & 3 & 5,66 & 3 & 5,66 & 6 & 11,32 & \multirow[t]{4}{*}{0,60} \\
\hline & Betametildigoxina & 0 & 0 & 1 & 1,89 & 1 & 1,89 & \\
\hline & Digoxina & 0 & 0 & 1 & 1,89 & 1 & 1,89 & \\
\hline & $\begin{array}{l}\text { No tiene } \\
\text { tratamiento }\end{array}$ & 22 & 41,51 & 23 & 43,40 & 45 & 84,91 & \\
\hline \multirow{2}{*}{$\begin{array}{l}\text { Tratamiento } \\
\text { anticoagulante }\end{array}$} & $\mathrm{Si}$ & 19 & 35,85 & 17 & 32,08 & 36 & 67,92 & \multirow[t]{2}{*}{0,23} \\
\hline & No & 6 & 11,32 & 11 & 20,75 & 17 & 32,08 & \\
\hline \multirow{5}{*}{$\begin{array}{l}\text { Medicamento } \\
\text { Anticoagulante }\end{array}$} & Rivaroxaban & 16 & 30,19 & 11 & 20,75 & 27 & 50,94 & \multirow[t]{5}{*}{0,60} \\
\hline & Warfarina & 3 & 5,66 & 1 & 1,89 & 4 & 7,55 & \\
\hline & Dabigatrán & 0 & 0 & 3 & 5,66 & 3 & 5,66 & \\
\hline & Apixaban & 0 & 0 & 2 & 3,77 & 2 & 3,77 & \\
\hline & $\begin{array}{l}\text { No tiene } \\
\text { tratamiento }\end{array}$ & 6 & 11,32 & 11 & 20,75 & 17 & 32,08 & \\
\hline
\end{tabular}

Tabla 3. Variables de la escala CHA2-DS2-VASC por sexo.

\begin{tabular}{|c|c|c|c|c|c|c|c|c|}
\hline \multirow[t]{2}{*}{ Variable } & \multirow[t]{2}{*}{ Categorías } & \multicolumn{2}{|l|}{ Masculino } & \multicolumn{2}{|l|}{ Femenino } & \multicolumn{2}{|l|}{ Total } & \multirow{2}{*}{$\begin{array}{lr}\text { Valor } & \text { de } \\
\text { p } & \text { (Chi } \\
\text { cuadrado) }\end{array}$} \\
\hline & & $\begin{array}{l}\text { Numero } \\
\text { n: } 25\end{array}$ & $\begin{array}{l}\text { Porcentaje } \\
\%\end{array}$ & $\begin{array}{l}\text { Num ero } \\
n: 28\end{array}$ & $\begin{array}{l}\text { Porcentaje } \\
\%\end{array}$ & $\begin{array}{l}\text { Numero } \\
\text { n: } 53\end{array}$ & $\begin{array}{l}\text { Porcentaje } \\
\%\end{array}$ & \\
\hline \multirow[t]{2}{*}{$\begin{array}{l}\text { Escala CHA2- } \\
\text { DS2-VASC }\end{array}$} & $\begin{array}{l}\text { Presente en la } \\
\text { historia clínica }\end{array}$ & 25 & 47,17 & 28 & 52,83 & 53 & 100 & \\
\hline & $\begin{array}{l}\text { Ausente en la } \\
\text { historia clínica }\end{array}$ & 0 & 0 & 0 & 0 & 0 & 0 & \\
\hline \multirow[t]{2}{*}{ Falla Cardíaca } & No & 19 & 35,85 & 20 & 37,74 & 39 & 73,58 & \multirow[t]{2}{*}{0,60} \\
\hline & $\mathrm{Si}$ & 6 & 11,32 & 8 & 15,09 & 14 & 26,42 & \\
\hline \multirow[t]{2}{*}{ Hipertensión } & No & 0 & 0 & 0 & 0 & 0 & 0 & \\
\hline & Sí & 25 & 47,17 & 28 & 52,83 & 53 & 100 & \\
\hline \multirow[t]{2}{*}{ Edad $\geq 75$ años } & No & 9 & 16,98 & 8 & 15,09 & 17 & 32,08 & \multirow[t]{2}{*}{0,56} \\
\hline & Sí & 16 & 30,19 & 20 & 37,74 & 36 & 67,92 & \\
\hline \multirow[t]{2}{*}{ Diabetes } & No & 14 & 26,42 & 19 & 35,85 & 33 & 62,26 & \multirow[t]{2}{*}{0,37} \\
\hline & Sí & 11 & 20,75 & 9 & 16,98 & 20 & 37,74 & \\
\hline \multirow[t]{2}{*}{$\mathrm{ACV}$} & No & 23 & 43,40 & 26 & 49,06 & 49 & 92,45 & \multirow[t]{2}{*}{0,90} \\
\hline & Sí & 2 & 3,77 & 2 & 3,77 & 4 & 7,55 & \\
\hline \multirow{2}{*}{$\begin{array}{l}\text { Enfermedad } \\
\text { Vascular }\end{array}$} & No & 17 & 32,08 & 19 & 35,85 & 36 & 67,92 & \multirow[t]{2}{*}{0,99} \\
\hline & Sí & 8 & 15,09 & 9 & 16,98 & 17 & 32,08 & \\
\hline \multirow{2}{*}{$\begin{array}{l}\text { Edad de } 65 \text { a } \\
75\end{array}$} & No & 17 & 32,08 & 21 & 39,62 & 38 & 71,70 & \multirow[t]{2}{*}{0,57} \\
\hline & Sí & 8 & 15,09 & 7 & 13,21 & 15 & 28,30 & \\
\hline \multirow{3}{*}{$\begin{array}{l}\text { Clasificación } \\
\text { del riesgo }\end{array}$} & Leve & 0 & 0 & 0 & 0 & 0 & 0 & \multirow[t]{3}{*}{0,05} \\
\hline & Moderado & 5 & 9,43 & 1 & 1,89 & 6 & 11,32 & \\
\hline & Severo & 20 & 37,74 & 27 & 50,94 & 47 & 88,68 & \\
\hline
\end{tabular}


Tabla 4. Escala CHA2-DS2-VASC por diagnóstico de ACV.

\begin{tabular}{|c|c|c|c|c|c|c|c|c|}
\hline \multicolumn{2}{|c|}{ Variables categóricas } & \multicolumn{7}{|c|}{ Diagnóstico de ACV } \\
\hline \multirow[t]{2}{*}{ Variable } & \multirow[t]{2}{*}{ Categorías } & \multicolumn{2}{|c|}{ Sí } & \multicolumn{2}{|l|}{ No } & \multicolumn{2}{|l|}{ Total } & \multirow{2}{*}{$\begin{array}{l}\text { P } \quad \text { (Chi } \\
\text { cuadrado) }\end{array}$} \\
\hline & & $\begin{array}{l}\text { Numero n } \\
: 4\end{array}$ & $\begin{array}{l}\text { Porcentaje } \\
\%\end{array}$ & $\begin{array}{l}\text { Numero } \\
\text { n: } 49\end{array}$ & $\begin{array}{l}\text { Porcentaje } \\
\%\end{array}$ & $\begin{array}{l}\text { N u m e ro } \\
\text { n: } 53\end{array}$ & $\begin{array}{l}\text { Porcentaje } \\
\%\end{array}$ & \\
\hline \multirow{2}{*}{$\begin{array}{l}\text { F a l l a } \\
\text { Cardíaca }\end{array}$} & No & 3 & 5,66 & 36 & 67,92 & 39 & 73,58 & \multirow[t]{2}{*}{0,94} \\
\hline & $\mathrm{Si}$ & 1 & 1,89 & 13 & 24,53 & 14 & 26,42 & \\
\hline \multirow{2}{*}{$\begin{array}{l}\text { Edad } \geq \\
\text { años }\end{array}$} & No & 1 & 1,89 & 16 & 30,19 & 17 & 32,08 & \multirow[t]{2}{*}{0,75} \\
\hline & Sí & 3 & 5,66 & 33 & 62,26 & 36 & 67,92 & \\
\hline \multirow[t]{2}{*}{ Diabetes } & No & 2 & 3,77 & 31 & 58,49 & 33 & 62,26 & \multirow[t]{2}{*}{0,59} \\
\hline & Sí & 2 & 3,77 & 18 & 33,96 & 20 & 37,74 & \\
\hline \multirow{2}{*}{$\begin{array}{l}\text { Enfermedad } \\
\text { Vascular }\end{array}$} & No & 0 & 0 & 36 & 67,92 & 36 & 67,92 & \multirow[t]{2}{*}{$<0,01$} \\
\hline & Sí & 4 & 7,55 & 13 & 24,53 & 17 & 32,08 & \\
\hline \multirow{2}{*}{$\begin{array}{l}\text { Edad de } 65 \text { a } \\
75 \text { años }\end{array}$} & $\mathrm{No}$ & 3 & 5,66 & 35 & 66,04 & 38 & 71,70 & \multirow[t]{2}{*}{0,87} \\
\hline & Sí & 1 & 1,89 & 14 & 26,42 & 15 & 28,30 & \\
\hline \multirow{2}{*}{$\begin{array}{l}S \quad \text { e } x \quad o \\
\text { femenino }\end{array}$} & $\mathrm{No}$ & 2 & 3,77 & 23 & 43,40 & 25 & 47,27 & \multirow[t]{2}{*}{0,90} \\
\hline & $\mathrm{Si}$ & 2 & 3,77 & 26 & 49,06 & 28 & 52,83 & \\
\hline \multirow{3}{*}{$\begin{array}{l}\text { Clasificación } \\
\text { del riesgo }\end{array}$} & Leve & 0 & 0 & 0 & 0 & 0 & 0 & \multirow[t]{3}{*}{0,45} \\
\hline & Moderado & 0 & 0 & 6 & 11,32 & 6 & 11,32 & \\
\hline & Severo & 4 & 7,55 & 43 & 81,13 & 47 & 88,68 & \\
\hline
\end{tabular}

